\title{
Integração do processo de ajuste de histórico com a modelagem geoestatística em reservatórios de petróleo
}

\author{
Célio Maschio ${ }^{I}$, Alexandre Campane Vidal ${ }^{2}$ \& Denis José Schiozer ${ }^{I}$
}

\begin{abstract}
Resumo O processo de ajuste de histórico de produção, onde o modelo numérico é calibrado para reproduzir a produção real do campo, normalmente é realizado de forma separada da modelagem geológica. Geralmente, a construção do modelo geológico e o processo de ajuste são realizados por equipes diferentes, sendo comum um desacoplamento ou um acoplamento fraco entre as duas áreas. Isso pode levar, na fase do ajuste, a alterações inadequadas no modelo geológico, podendo resultar em modelos geologicamente inconsistentes. Este trabalho propõe uma integração entre a modelagem geoestatística e o ajuste de histórico através da incorporação das realizações geoestatísticas ao processo de ajuste de histórico assistido. Desta forma, são considerados no ajuste, os parâmetros de reservatório como, por exemplo, propriedades de interação entre rocha e fluido, bem como as imagens geradas pelas realizações geoestatísticas. Para encontrar as melhores combinações dos parâmetros que melhor ajustam o histórico, um método de otimização baseado em algoritmo genético é utilizado. A metodologia proposta é aplicada a um modelo sintético com características reais de reservatórios de petróleo. O ajuste de histórico é realizado de forma convencional e considerando as imagens geoestatísticas como parâmetros de ajuste, sendo, posteriormente, comparados os dois processos. Os resultados mostram a viabilidade e as vantagens decorrentes deste processo de integração entre o ajuste de histórico e a modelagem geoestatística.
\end{abstract}

Palavras-chave: ajuste de histórico, simulação de reservatórios, geoestatística.

\begin{abstract}
Integration of the history matching process with the geostatistical modeling in petroleum reservoirs. The production history matching process, by which the numerical model is calibrated in order to reproduce the observed field production, is normally carried out separately from the geological modeling. Generally, the construction of the geological model and the history matching process are performed by different teams, such is common uncoupling or a weak coupling between the two areas. This can lead, in the history matching step, inadequate changes in the geological model, resulting sometimes models geologically inconsistent. This work proposes integration between the geostatistical modeling and the history matching through the incorporation of geostatistical realizations into the assisted process. In this way, reservoir parameters such as rock-fluid interaction properties, as well as the images resulted from the realizations are considered in the history matching. In order to find the best parameters combination that adjusts the model to the observed data, an optimization routine based on genetic algorithm is used. The proposed methodology is applied to a synthetic realistic reservoir model. The history matching is carried out in the conventional manner and considering the geostatistical images as history parameters, such the two processes are posteriorly compared. The results show the feasibility and the advantages resulting of this process of integration between the history matching and geostatistical modeling.
\end{abstract}

Keywords: history matching, reservoir simulation, geostatistic.

\section{INTRODUÇÃO}

Ajuste de histórico de produção O ajuste de histórico de produção é um processo de calibração de um modelo de simulação através do aperfeiçoamento da caracterização do reservatório. É uma das tarefas mais realizadas pelo profissional ligado à simulação de reservatórios e ao gerenciamento de campos de petróleo. $\mathrm{O}$ ajuste de histórico é necessário, pois devido (1) às incertezas presentes no reservatório, (2) ao alto custo das informações e (3) perda de informações na passagem dos dados da escala geológica para e escala de simulação, o modelo de simulação concebido na fase de caracterização de reservatórios normalmente apresenta grandes limitações para previsão de produção.

Normalmente, o ajuste de histórico é realizado em uma etapa posterior à etapa de modelagem do reservatório. O modelo gerado pela equipe de geologia á passado para a equipe de engenharia que passa a realizar alterações diretamente no modelo geológico, através de multiplicadores de porosidade e permeabilidade, por exemplo.

O ajuste de histórico pode ser realizado de forma manual ou assistido. $\mathrm{O}$ ajuste manual se caracteriza

1 - Depto. de Engenharia de Petróleo - Faculdade de Engenharia Mecânica - UNICAMP Campinas (SP), Paulo, Brasil. E-mails: celio@dep.fem.unicamp.br, denis@dep.fem.unicamp.br

2 - Depto. de Geologia e Recursos Naturais - Instituto de Geociências - UNICAMP Campinas (SP), Brasil. E-mail: vidal@ige.unicamp.br 
por ser um processo de tentativa e erro. Normalmente, é mais trabalhoso do ponto de vista de esforço do profissional envolvido no processo, pois todas as tarefas, tais como alterações do modelo de simulação, execução das simulações, análise dos resultados, dentre outras, são realizadas de forma manual e exigem mais interação com o processo, tomando mais tempo de trabalho. $\mathrm{O}$ ajuste assistido consiste em automatizar as tarefas manuais. Além disso, para se encontrar a melhor combinação de parâmetros (propriedades do modelo a serem alteradas) que minimize a função-objetivo (diferença entre os dados observados e os dados simulados) utiliza-se um algoritmo de otimização. O processo de ajuste assistido tem como principais metas ser menos trabalhoso, por automatizar partes do processo, e ao mesmo tempo ser mais confiável, por investigar melhor o espaço de soluções. A idéia é também aproveitar a experiência do profissional permitindo a interação com o processo, podendo realizar o ajuste por etapas.

Ajuste de histórico e modelagem geoestatística $\mathrm{Al}$ guns trabalhos encontrados na literatura abordam o tema da combinação do ajuste de histórico com a modelagem geoestatística. Porém, o processo é realizado em etapas separadas. Benett \& Graft 2002 propuseram uma metodologia para integrar uma ferramenta de ajuste de histórico baseado em cálculo de gradientes com o ajuste de diversos cenários geológicos. Uma das características da metodologia era a seleção e descarte de modelos de acordo com a diferença entre os dados simulados e os dados observados. O processo consistia em gerar múltiplas realizações e realizar o ajuste em modelo de reservatório resultante de cada uma das realizações, usando parâmetros determinísticos do reservatório, como por exemplo, permeabilidade relativa do sistema rocha-fluido.

Tureyen \& Caers 2003 propuseram uma metodologia para otimização em dois níveis, considerando um nível em escala geológica e outro em escala de simulação. Gross et al. (2004) apresentaram um método baseado em simulação por linhas de corrente (streamline simulation) combinado com a simulação seqüencial geoestatística. O uso da simulação por linhas de corrente combinado com modelos em escala geológica gerados por técnicas geoestatísticas também foi abordado por Le Ravalec-Dupin e Fenwick (2002).

Caers (2002) abordou o ajuste de histórico combinado com a modelagem geoestatística através do uso da técnica da cadeia de Markov. Caers (2003) apresentou um método de deformação gradual combinando também com a simulação por linhas de corrente. Hoffman e Caers (2005) e Hoffman e Caers (2006) desenvolveram um método de perturbação regional do modelo usando modelagem geoestatística para ajuste de histórico. Suzuki e Caers (2006) apresentaram um método de busca estocástica para encontrar modelos geologicamente consistentes baseados na comparação com dados observados (histórico). Apesar de não terem abordado o tema, Kashib \& Srinivasan (2006) afirmam que a influência do modelo de variograma nos resulta- dos do ajuste de histórico é um importante tópico de pesquisa.

Algoritmo genético $\mathrm{O}$ uso das imagens geoestatísticas como parâmetros de ajuste gera uma dificuldade para os algoritmos de otimização normalmente empregados no ajuste de histórico assistido. Tradicionalmente, os métodos empregados no processo de ajuste de histórico são métodos que, de alguma forma dependem de uma direção de descida em um espaço de soluções (combinação dos parâmetros de ajuste). Esses métodos geralmente falham em problemas altamente não-lineares, ou seja, problemas onde não há uma relação direta (linearidade) entre as variáveis de entrada (parâmetros de ajuste) e a variável de saída (função objetivo).

Os algoritmos genéticos (Velez-Langs 2005, Romero \& Carter 2001) são métodos de otimização baseados em processos de evolução natural. Uma das características que torna estes métodos interessantes e atrativos é a habilidade em lidar com problemas altamente não lineares, com espaços de soluções extremamente irregulares e não suaves, onde os métodos tradicionais, que dependem de uma direção de descida (informações de gradientes), geralmente falham. Outra característica interessante é facilidade de usar a computação distribuída.

Os algoritmos genéticos operam pela definição inicial de uma geração com um número $\mathrm{N}$ de indivíduos, sendo que cada indivíduo é avaliado de acordo com o valor da função objetivo. Há três tipos básicos de operadores: seleção (ou reprodução), também conhecido como elitismo, mutação e crossover. Principalmente estes dois últimos exercem influência significativa nos resultados da otimização. É necessário definir valores adequados para eles de tal forma que fique equilibrado o aspecto estocástico e determinístico que caracterizam o método.

METODOLOGIA A metodologia proposta neste trabalho consiste na integração entre a modelagem geoestatística e o ajuste de histórico através da incorporação das realizações geoestatísticas ao processo de ajuste de histórico assistido. A primeira etapa do processo é a caracterização e a geração do modelo geológico, que compreende a geração das litofacies e das propriedades petrofísicas como, por exemplo, porosidade e permeabilidade. Estas propriedades, incluindo a distribuição de litofacies, são usadas como parâmetros no processo de ajuste.

A figura 1 apresenta um fluxograma geral da metodologia proposta. Inicialmente, um conjunto de parâmetros, que pode incluir outros parâmetros além daqueles relacionados às realizações geoestatísticas e a função objetivo são definidos. Um método de otimização é então iniciado (neste caso o algoritmo genético). As propriedades petrofísicas são mapeadas dentro das litofacies para preenchimento da malha que representa o reservatório. Este mapeamento é feito durante o processo de otimização de forma automática. Em seguida realiza-se um processo de transferência de escala para 


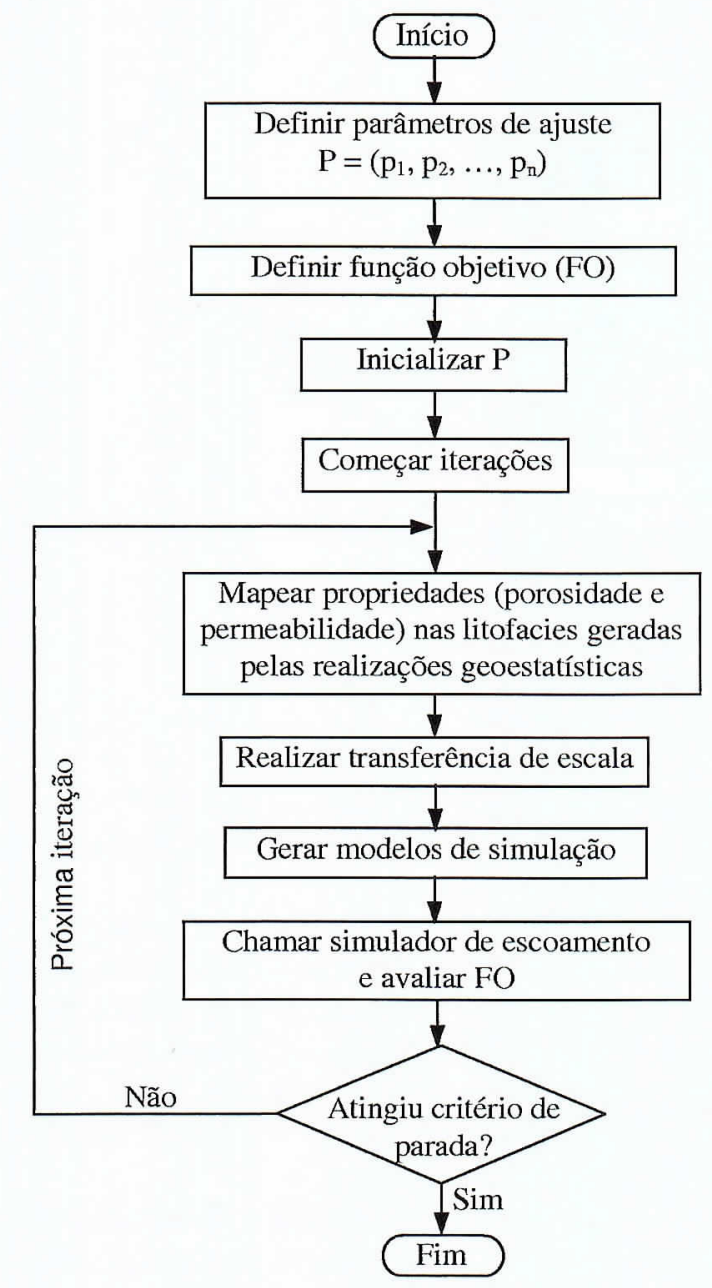

Figura 1 - Fluxograma geral da metodologia.

redução do tamanho da malha para adaptação ao modelo de simulação. Após este passo, os modelos de simulação são gerados e submetidos ao simulador de escoamento. Após a simulação, a função objetivo é avaliada e este processo iterativo continua até que o critério de parada seja atingido.

Transferência de escala Na prática da indústria do petróleo, os modelos geológicos são gerados em uma escala diferente das escalas usada nos simuladores de escoamento. O modelo geológico é construído em uma escala bem refinada, gerando milhões de células na representação do reservatório. Por outro lado, os simuladores comerciais comportam modelos da ordem de uma a três centenas de milhares de células. Por isso, é necessário um processo de mudança de escala do modelo geológico para tornar viável a simulação de escoamento.

Um procedimento de transferência de escala foi integrado ao processo de ajuste de histórico. Um programa de transferência de escala foi integrado ao programa de ajuste de histórico para ser executado de forma automática. Os detalhes do método utilizado podem ser encontrados em Maschio \& Schiozer (2003). Para a transferência de escala da permeabilidade absoluta foi utilizada a equação 1 :

$$
K_{e q=\left(C_{\min } C_{\max }\right)^{n}}
$$

onde Keq é a permeabilidade equivalente, Cmin e Cmax são os limites de Cardwell e Parsons. Detalhes sobre estes limites podem vistos nos trabalhos de Renard e Marsily (1997) e Renard et al. (2000).

Cálculo da função objetivo A função objetivo, que expressa matematicamente a diferença entre os dados simulados e observados, é calculada de acordo com a equacão 2:

$$
O F=\sqrt{\frac{\sum_{j=1}^{M} w_{j} \sum_{i=1}^{N_{j}}\left(d_{o b s}^{i j}-d_{s i m}^{i j}\right)^{2}}{m}}
$$

onde $\mathrm{M}$ é o número de série de dados (por exemplo, dados de pressão e produção dos poços), $w_{j}$ são pesos $d_{o b s}^{i j} \quad d_{s i m}^{i j}$ atribuídos à série de dados, $\mathrm{N}_{\mathrm{j}}$ é o núme$r \quad o$ de dados observados de cada série de dados, e são os dados observados e simulados, respectivamente e $\mathrm{m}$ é o total de dados observados.

\section{APLICAÇÃO}

Características gerais do modelo de reservatório A geração do modelo de reservatório foi baseada nas informações de dez poços de um campo de petróleo. A descrição dos poços apresenta a composição de arenitos e folhelhos e para a identificação dessas litologias foram utilizados os perfis raios gama e densidade. A distribuição das facies foi feita pela simulação seqüencial da indicatriz com a geração de 200 modelos de fácies.

A atribuição dos valores de porosidade e permeabilidade foi realizada por meio de simulação estocástica não-condicional. Para isso foi utilizado o mesmo modelo de variograma da simulação de fácies e a distribuição dos valores para cada variável. A porosidade apresenta valor mínimo de $15 \%$ e máximo de $35 \%$, com média de $25 \%$, e para a variável permeabilidade o valor médio adotado foi de $1250 \mathrm{mD}$, com mínimo de $500 \mathrm{mD}$ e máximo de $2000 \mathrm{mD}$. O método geoestatístico aplicado foi da simulação seqüencial gaussiana. Foram gerados 50 modelos de porosidade e 50 modelos de permeabilidade.

As propriedades petrofisicas geradas foram inseridas apenas para as células do grid definidas como arenitos, enquanto para as facies não-reservatório foram adotados valores constantes de $5 \mathrm{mD}$ para permeabilidade e $5 \%$ para porosidade.

$\mathrm{O}$ modelo geológico do reservatório foi discretizado em uma malha com 50 blocos na direção x, 50 blocos na direção y e 46 blocos na direção z (46 camadas), com dimensões de $80 \mathrm{~m}, 60 \mathrm{~m}$ e $2 \mathrm{~m}$, respectivamente. Uma visão tridimensional do reservatório aparece na figura 2, onde a cor azul indica facies folhelho e as outras cores indicam facies arenito. Podem ser vistos dois 


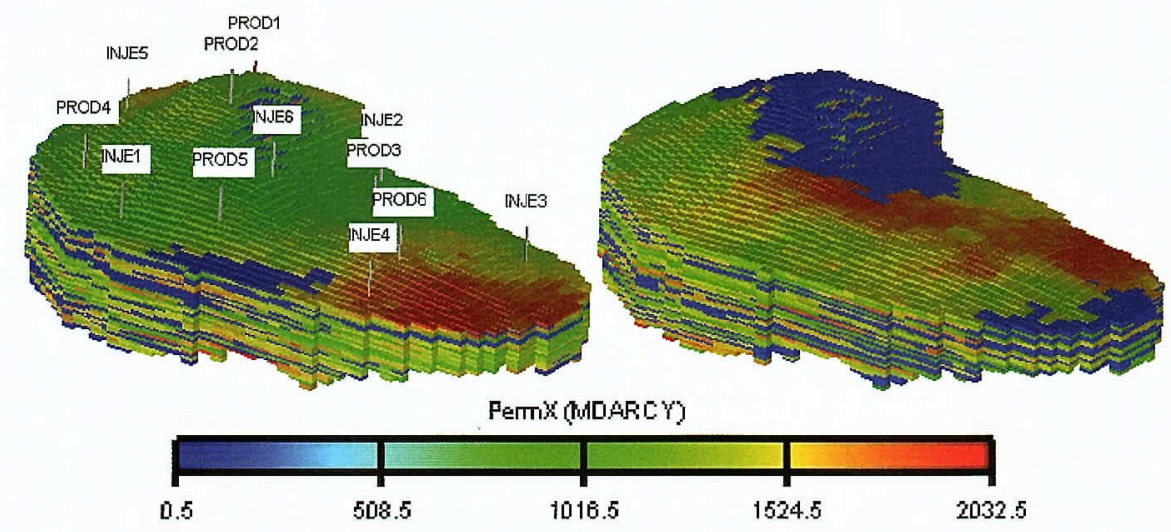

Figura 2 - Modelo do reservatório mostrando distribuição de facies $e$ permeabilidade: cor azul indica folhelhos e as outras cores indicam arenitos.

exemplos de reservatório, mostrando a distribuição de facies e o preenchimento das mesmas com os valores de permeabilidade. Pode-se ver também a localização dos poços usados no esquema de produção do reservatório, além dos 6 poços usados na modelagem, foram adicionados mais 6 . Do total de 12 poços, 6 são produtores e 6 injetores de água. O modelo de fluido usado foi do tipo Black-Oil e o modelo de permeabilidade relativa usado foi um modelo teórico do tipo Corey. O modelo geológico de referência foi simulado para gerar um histórico de 20 anos, sendo 10 anos usados para o ajuste de histórico e os 10 anos restantes para uma análise posterior de previsão de produção dos modelos ajustados, para efeito de validação e comparação dos processos de ajuste analisados neste trabalho. Durante o processo de ajuste, o número de blocos para o modelo simplificado, ou seja, com simplificação de escala, foi reduzido para 25 na direção x e 25 na direção y, gerando modelos com $25 \times 25 \times 46$ blocos.

Ajuste A - Ajuste convencional $\mathrm{O}$ ajuste A corresponde à realização do ajuste de forma convencional, ou seja, alterando diretamente o modelo geológico simplificado através de multiplicadores de porosidade e permeabilidade. Na tabela 1, é apresentada a descrição dos parâmetros de ajuste utilizados no Caso A. Além da alteração da porosidade e permeabilidade, são alterados também as curvas de permeabilidade relativa à água nas duas facies presentes no reservatório e o expoente $n$ relativo à transferência de escala (equação 1 ). $\mathrm{O}$ expoente $\mathrm{n}$ tem um considerável impacto no resultado da transferência de escala que, por sua vez, impacta no resultado da simulação. Por esse motivo, esse expoente foi incluído como uma variável no processo de ajuste.

Ajuste B - Ajuste utilizando modelos gerados por geoestatística $\mathrm{O}$ ajuste $\mathrm{B}$ corresponde à realização do ajuste utilizando os modelos geológicos gerados por geoestatística. Além dos três parâmetros correspondentes às realizações geoestatísticas (200 imagens de litofacies, 50 de porosidade e 50 de permeabilidade), são considerados também o parâmetro relacionado à trans- ferência de escala (n) e a razão entre a permeabilidade vertical e horizontal (KZ/KX). A descrição dos parâmetros aparece na tabela 2 .

Em ambos os casos de ajuste, a função objetivo foi composta pela vazão de água e pala pressão de fundo dos 6 poços produtores, de acordo com a equação 2. Como no Ajuste $\mathrm{B}$ existe o parâmetro relacionado à distribuição de facies e não existe um equivalente no Ajuste A, foi escolhida a permeabilidade relativa (de ambas as facies) para o Ajuste A.

O objetivo do ajuste de histórico é obter modelos de reservatórios de petróleo mais confiáveis para a previsão de produção, buscando melhorar a qualidade do processo decisório. Desta forma, os modelos de reservatório ajustados foram extrapolados por um período adicional de 10 anos para comparar a previsão de produção dos modelos obtidos com o Ajuste A e Ajuste B, e comparar também com os resultados da previsão do modelo geológico de referência. $\mathrm{Na}$ fase de previsão, os poços produtores foram controlados por pressão, ou seja, foi definida uma pressão mínima de fundo para os poços produtores e uma pressão de injeção máxima para os poços injetores.

RESULTADOS E DISCUSSÕES Nas tabelas 3 e 4, são mostrados os valores finais dos parâmetros do ajuste convencional (Ajuste A) e do ajuste considerando as realizações geoestatísticas (Ajuste B), respectivamente. Pode-se notar que no ajuste A, três dos seis parâmetros (multiplicadores de porosidade, permeabilidade e relação entre permeabilidade vertical e horizontal) assumiram os valores extremos mínimos da faixa de variação definidos na tabela 1 . Isso sugere que estes extremos poderiam se redimensionados, porém a definição de valores muito baixos para esses multiplicadores pode levar a valores irreais das propriedades. Em relação ao Ajuste B, exceto a porosidade, todos ou outros parâmetros assumiram valores intermediários em relação à faixa definida na tabela 2. Porém, o fato do parâmetro porosidade ter assumido o valor 50, não tem o mesmo significado quando comparado à situação de extremo no caso do Ajuste A, ou seja, seria possível continuar 
Tabela 1 - Descrição dos parâmetros de ajuste para o Caso A.

\begin{tabular}{|c|c|c|c|c|c|c|}
\hline & Porosidade & $\begin{array}{c}\text { Permeabilidade } \\
\text { horizontal }\end{array}$ & Razão KZ/KX & nKRA & $\mathrm{nKRF}$ & $\begin{array}{l}\text { Expoente n } \\
\text { (Equação 1) }\end{array}$ \\
\hline Tipo & \multicolumn{3}{|c|}{ Multiplicador } & \multicolumn{2}{|c|}{ Permeabilidade relativa } & $\begin{array}{c}\text { Transferência } \\
\text { de escala }\end{array}$ \\
\hline Mínimo & 0.85 & 0.50 & 0.05 & 1 & 1 & 0.45 \\
\hline Máximo & 1.15 & 2.00 & 0.50 & 4 & 4 & 0.55 \\
\hline Intervalos & 14 & 14 & 14 & 6 & 6 & 14 \\
\hline
\end{tabular}

$\mathrm{nKRA}=$ expoente para a permeabilidade relativa à água do modelo de Corey na facies arenito

$\mathrm{nKRF}=$ expoente para a permeabilidade relativa à água do modelo de Corey na facies folhelho

$\mathrm{KX}=$ permeabilidade absoluta na direção horizontal; $\mathrm{KZ}$ = permeabilidade absoluta na direção vertical

Tabela 2 - Descrição dos parâmetros de ajuste para o Caso B.

\begin{tabular}{l|c|c|c|c|c}
\hline & Litofacies & Porosidade & Permeabilidade & Razão KZ/KX & $\begin{array}{c}\text { Expoente n } \\
\text { (Equação 1) }\end{array}$ \\
\hline Tipo & \multicolumn{3}{|c|}{ Seqüência de imagens resultantes das realizações } & Multiplicador & $\begin{array}{c}\text { Transferência } \\
\text { de escala }\end{array}$ \\
\hline Mínimo & 1 & 1 & 1 & 0.05 & 0.45 \\
\hline Máximo & 200 & 50 & 50 & 0.50 & 0.55 \\
\hline Intervalos & 199 & 49 & 49 & 14 & 14 \\
\hline
\end{tabular}

Tabela 3 - Valores finais dos parâmetros do ajuste (Ajuste A).

\begin{tabular}{c|c|c|c|c|c}
\hline Porosidade & $\begin{array}{c}\text { Permeabilidade } \\
\text { horizontal }\end{array}$ & Razão KZ/KX & nKRA & nKRF & $\begin{array}{c}\text { Expoente n } \\
\text { (equação 1) }\end{array}$ \\
\hline \multicolumn{5}{c}{ Multiplicador } \\
\hline 0.850 & 0.500 & 0.050 & Permeabilidade relativa & $\begin{array}{c}\text { Transferência de } \\
\text { escala }\end{array}$ \\
\hline
\end{tabular}

Tabela 4 - Valores finais dos parâmetros do ajuste utilizando os modelos gerados por geoestatística (Ajuste B).

\begin{tabular}{c|c|c|c|c}
\hline Litofacies & Porosidade & Permeabilidade & Razão KZ/KX & Expoente n (equação 1) \\
\hline \multicolumn{3}{|c|}{ Realização geoestatística } & Multiplicador & Transferência de escala \\
\hline 69 & 50 & 47 & 0.27 & 0.453 \\
\hline
\end{tabular}

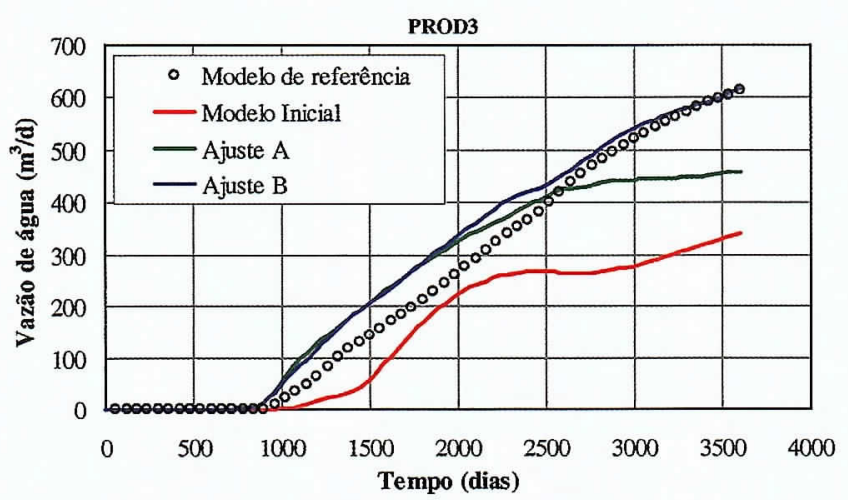

Figura 3 - Comparação dos processos de ajuste para a vazão de água do poço PROD3.

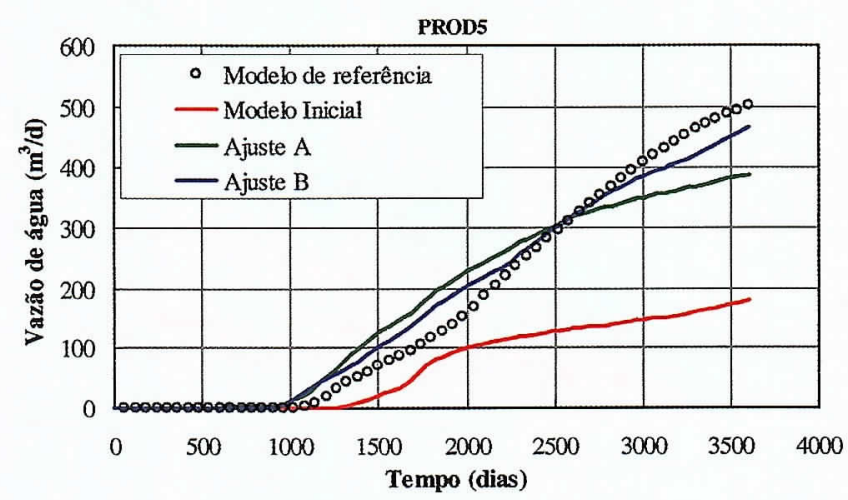

Figura 4 - Comparação dos processos de ajuste para a vazão de água do poço PROD5. 
gerando modelos de porosidade de forma que os valores continuariam no intervalo entre $15 \%$ e $35 \%$, conforme a premissa de construção do modelo.

Nas figuras 3, 4 e 5, são apresentados os gráficos com a comparação dos processos de ajuste para a vazão de água dos poços PROD3, PROD5 e PROD6, respectivamente. Pode-se observar que o ajuste B apresenta melhores resultados.

Na figura 6 é apresentado o gráfico de correlação entre os dados observados (com base na simulação do modelo geológico de referência) e os dados simulados para o modelo inicial, ajuste A e Ajuste B. Neste gráfico, estão todos os dados de todos os poços produtores constituindo, assim, em uma forma de análise global da qualidade do ajuste. Pontos mais próximos à reta a 45 graus indicam melhor qualidade do ajuste. Pode-se observar pelo gráfico que a qualidade do Ajuste $\mathrm{B}$ (ajuste utilizando modelos gerados por geoestatística) está melhor que o Ajuste A (ajuste convencional). Observa-se também que ainda seria possível melhorar o ajuste em ambos os casos (Ajuste A e Ajuste B) através de ajustes locais nas regiões dos poços, porém este não é o objetivo do presente trabalho.

Para o Ajuste A, foram usadas 469 simulações

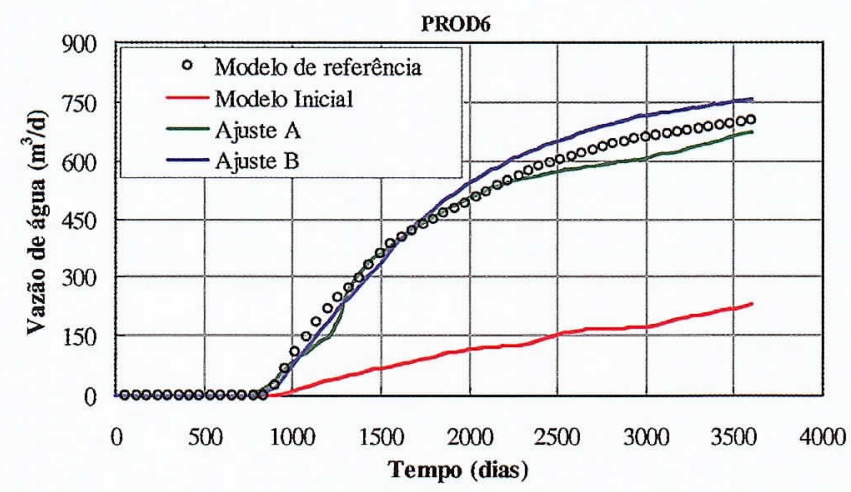

Figura 5 - Comparação dos processos de ajuste para a vazão de água do poço PROD6.

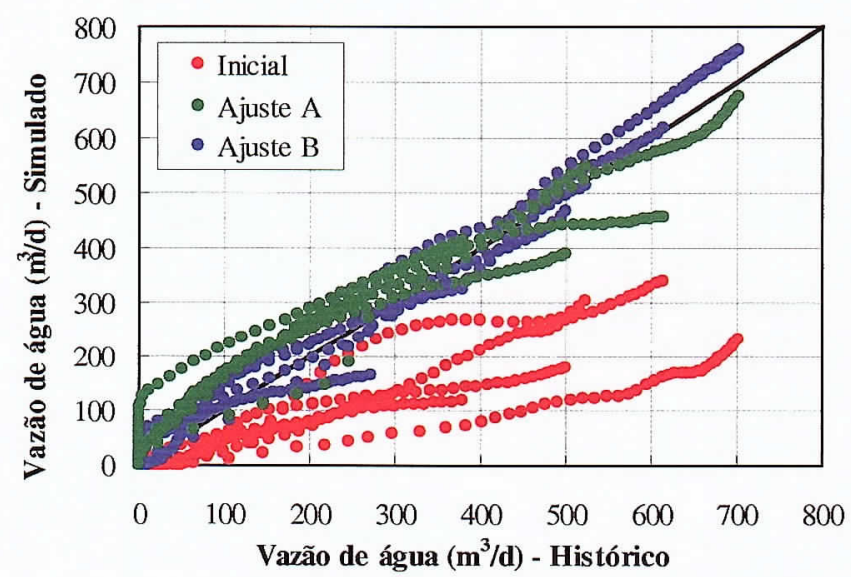

Figura 6 - Correlação entre dados observados e simulados para o modelo inicial, Ajuste A e Ajuste B. e, para o Ajuste B, 438 simulações. O número de simulações depende das configurações do algoritmo. No trabalho de Maschio et al. (2008), é apresentada uma análise dessas configurações e o impacto no número de simulações. Naquele trabalho, mostra-se que é possível reduzir o número de simulações sem perder a qualidade do ajuste.

A figura 7 mostra a extrapolação dos modelos com a previsão de produção acumulada de óleo do campo. Pode-se notar que a previsão de produção de óleo está bem próxima do histórico em ambos os modelos, significando que ambos apresentam previsão confiável

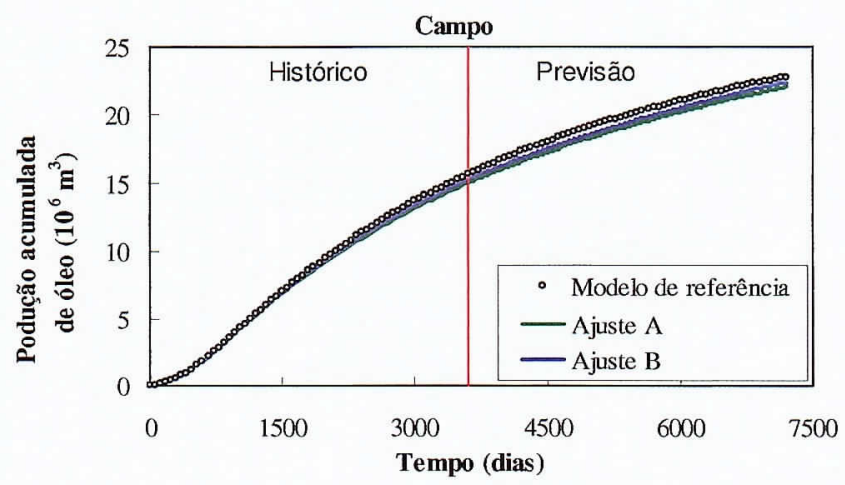

Figura 7 - Previsão da produção acumulada de óleo do campo.

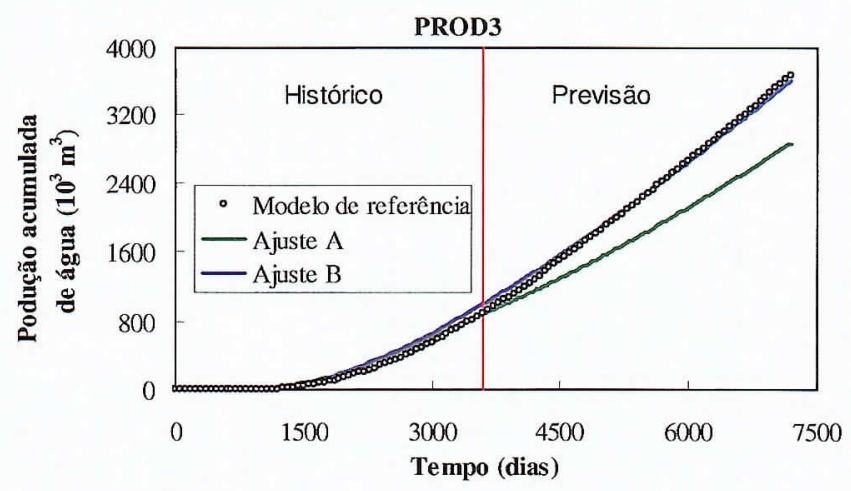

Figura 8 - Previsão da produção acumulada de água do poço produtor PROD3.

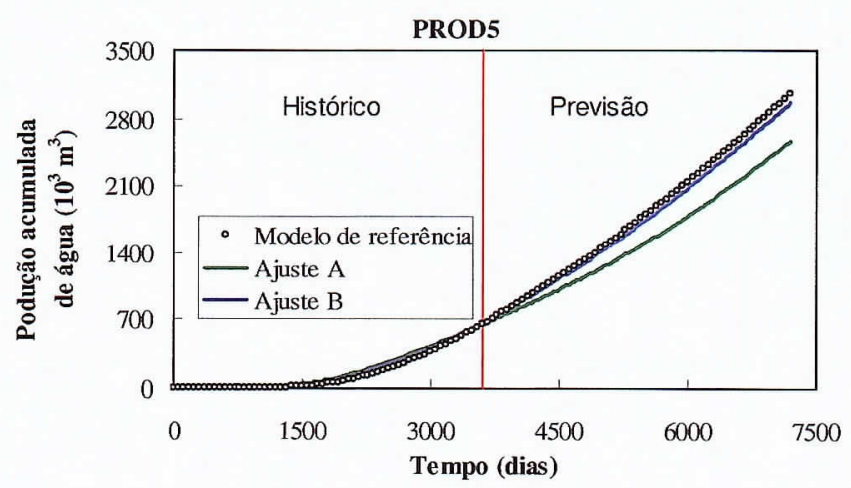

Figura 9 - Previsão da produção acumulada de água do poço produtor PROD5. 


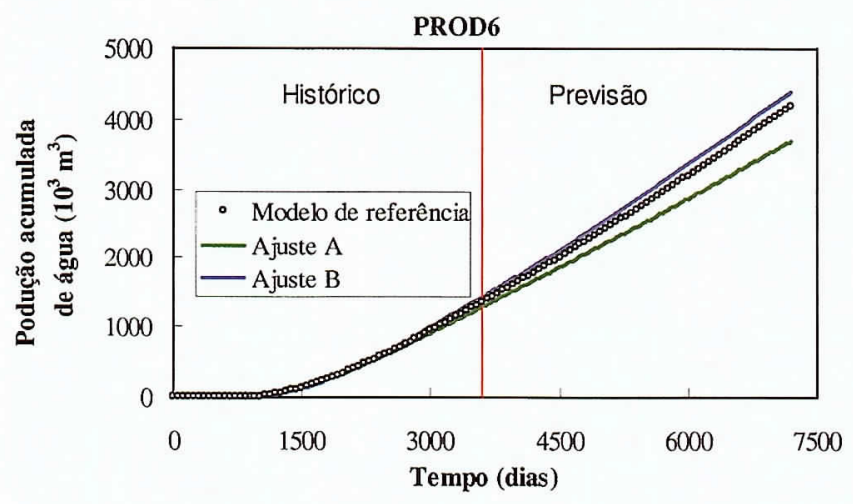

Figura 10 - Previsão da produção acumulada de água do poço produtor PROD6.

para produção de óleo. No entanto, o modelo obtido com o Ajuste B é mais confiável para previsão de produção de água do que o modelo obtido com o Ajuste A. Isso pode ser observado nas figuras 8,9 e 10 , que mostram a previsão da produção acumulada de água dos poços PROD3, PROD5 e PROD6, para ambos os modelos.

CONCLUSÕES Neste trabalho, foi apresentada uma metodologia para tratar imagens geradas por realizações geoestatísticas como parâmetros do processo de ajuste de histórico, visando integrar a modelagem geoestatística com o processo de ajuste. A metodologia foi comparada com o processo de ajuste convencional. Através dos resultados foi possível observar que a integração posposta pode fornecer melhores resultados no ajuste quando comparados com o método tradicional. Foi observado também que o modelo obtido com o Ajuste $\mathrm{B}$ resultou em previsões mais confiáveis do que o modelo obtido com o Ajuste A (ajuste convencional). A geração do modelo geológico durante o processo de ajuste permite manter as premissas originais, gerando modelos geologicamente mais consistentes.

Agradecimentos Os autores agradecem ao CEPETRO e a PETROBRAS pelo apoio financeiro.

\section{Referências}

Bennett F. \& Graf T. 2002. Use of Geostatistical Modeling and Automatic History Matching to Estimate Production Forecast Uncertainty - A Case Study (SPE 74389). In: SPE International Petroleum Conference and Exhibition in Mexico, Villahermosa, Mexico, CD-ROM.

Caers J. 2002. Geostatistical History Matching Under Training-Image Based Geological Model Constraints (SPE 77429). In: SPE Annual Technical Conference and Exhibition, San Antonio, Texas, CD-ROM.

Caers J. 2003. Efficient gradual deformation using a streamline-based proxy method. Journal of Petroleum Science and Engineering, 39(1-2):57-83.

Gross H., Thiele M.R., Alexa M.J., Caers J. , Kovscek A.R. 2005. Streamline-Based History Matching Using Geostatistical Constraints: Application to a Giant, Mature Carbonate Reservoir (SPE 90069). In: SPE Annual Technical Conference and Exhibition, Houston, Texas, CD-ROM.

Hoffman B., Caers J. 2005. Regional probability perturbations for history matching. Journal of Petroleum Science and Engineering, 46(1-2):53-71.

Hoffman B. \& Caers J. 2007. History matching by jointly perturbing local facies proportions and their spatial distribution: Application to a North Sea reservoir. Journal of Petroleum Science and Engineering, 57(34):257-272.

Kashib T. \& Srinivasan S. 2006. A probabilistic approach to integrating dynamic data in reservoir models. Journal of Petroleum Science and Engineering, 50(3-4):241-257.

Le Ravalec-Dupin M., Fenwick D.H. 2002. A Combined Geostatistical and Streamline-Based History Matching Procedure (SPE 77378). In: SPE Annual Technical Conference and Exhibition, San Antonio, Texas, CDROM.

Maschio C. \& Schiozer D.J. 2003. A new upscaling technique based on Dykstra-Parsons coefficient: evaluation with streamline reservoir simulation. Journal of Petroleum Science and Engineering, 40(1-2):27-36.

Maschio C., Vidal A.C., Schiozer D.J. 2008. A framework to integrate history matching and geostatistical modeling using genetic algorithm and direct search methods. Journal of Petroleum Science and Engineering (In press).

Renard Ph. \& Marsily G. 1997. Calculating equivalent permeability: a review. Advances in Water Resources, 20(5-6):253-278.

Renard P., Le Loc'h G., Ledoux E., de Marsily G. \& Mackay R. 2000. A fast algorithm for the estimation of the equivalent hydraulic conductivity of heterogeneous media. Water Resources Research, 36(12):3567-3580.

Romero C.E. \& Carter J.N. 2001. Using genetic algorithms for reservoir characterization. Journal of Petroleum Science and Engineering, 31(2-4):113-123.

Suzuki S. \& Caers J. 2006. History Matching With an Uncertain Geological Scenario (SPE 102154). In: SPE Annual Technical Conference and Exhibition, San Antonio, Texas, CD-ROM.

Tureyen O. I. \& Caers J. 2003. A Two-Level Optimization Method for Geostatistical Integration of Production Data on Non-Uniform Grids (SPE 84368). In: SPE Annual Technical Conference and Exhibition, Denver, Colorado, CD-ROM.

Velez-Langs O. 2005. Genetic algorithms in oil industry: An overview. Journal of Petroleum Science and Engineering, 47(1-2):15-22.

Manuscrito BR 04

Submetido em 21 de dezembro de 2007 Aceito em 10 de maio de 2008 